

\title{
Passions françaises et culture de Guerre froide
}

Joëlle Beurier

\section{To cite this version:}

Joëlle Beurier. Passions françaises et culture de Guerre froide. Sous la direction de Philippe Buton, Olivier Büttner, Michel Hastings. : La Guerre froide vue d'en bas, CNRS Editions, p. 223-236, 2014, 9782271078230. halshs-01262814

\section{HAL Id: halshs-01262814 https://shs.hal.science/halshs-01262814}

Submitted on 27 Jan 2016

HAL is a multi-disciplinary open access archive for the deposit and dissemination of scientific research documents, whether they are published or not. The documents may come from teaching and research institutions in France or abroad, or from public or private research centers.
L'archive ouverte pluridisciplinaire HAL, est destinée au dépôt et à la diffusion de documents scientifiques de niveau recherche, publiés ou non, émanant des établissements d'enseignement et de recherche français ou étrangers, des laboratoires publics ou privés. 


\section{Passions françaises et culture de Guerre froide}

Joëlle Beurier

Comment retrouver les pensées du peuple de France durant la Guerre froide ? Pour dire les choses autrement, comment décrire la Guerre froide par le bas quand les sources sont majoritairement diplomatiques et renvoient largement au point de vue des grands de ce monde ? La presse illustrée est une entrée tout à fait passionnante dans l'imaginaire d'une nation, dans la mesure où, largement lue par les citoyens en complément de la presse écrite, elle ajoute aux faits et à leur analyse, la mise en images du discours. Par ailleurs, elle s'affiche comme un outil à la charnière entre l'information événementielle et une forme de distraction du public, créant pour cela le langage spécifique du photojournalisme, qui informe tout en jouant sur les émotions. En 1962, Paris-Match comme tous les magazines illustrés, est lu collectivement, en famille ou dans les lieux semi-publiques (coiffeur, professions médicales), circulant dans des cercles bien plus larges que celui des abonnés ou des lecteurs réguliers. Bien qu’en régression depuis 1960 - il tirait en 1958 à 1800000 exemplaires - son succès tient au fait que l'image d'actualité est encore rare : ce n'est qu'au début des années 1960 que la télévision commence son entrée de masse dans les foyers, et il faut attendre une décennie pour que sa généralisation soit enfin avérée. Voir l'actualité passe donc par la photographie, support essentiel de la presse illustrée, dont Paris-Match constitue l'acteur principal en France.

Mais peut-on considérer que le magazine illustré véhicule une vision vue du bas sur l'information qu'il propose ? En tant que vecteur principal d'une information visuelle essentiellement accessible par ses reportages, le magazine doit trouver un juste milieu entre la dimension factuelle de l'événement et les attentes de son lectorat. Depuis 1949, date de sa reparution après la Seconde Guerre mondiale, Jean Prouvost accorde à l'image une place essentielle entre narration factuelle et émotionnelle ${ }^{1}$. Par sa vocation généraliste, le magazine

1. Blandin Claire, «L’image au cœur de l'entreprise de presse de Jean Prouvost », in Beurier Joëlle, Taveaux-Grandpierre Karine (dir.), Le photojournalisme, de 1930 à nos jours, Rennes, Presses universitaires de Rennes, 2014, p. 71-81. 
aborde tous les sujets, pour faire entrer dans les chaumières l'actualité de la planète comme les affaires intérieures, jonglant avec le fait divers et l'actualité artistique avec la même faconde, élargissant ainsi aux centres d'intérêt les plus variés, l'information qu'il donne à voir au public, cherchant à rester en prises avec les passions françaises sous toutes leurs formes. À ce titre, il offre une entrée sur les représentations les plus collectives de la Guerre froide.

L’année 1962 est idéale pour aborder les rapports entre le plus célèbre illustré français et la Guerre froide. En effet, elle est particulièrement riche d'une actualité liée à cet événement puisque, malgré une détente relative depuis l'arrivée de Nikita Khrouchtchev aux commandes de l'URSS en 1956, on atteint, entre la crise de Berlin en août 1961 et la crise des fusées en octobre 1962, un apogée dans l'opposition Est-Ouest. 1962 est donc une bonne entrée sur la manière dont la population française vit la Guerre froide. Pour la sonder, nous nous concentrerons sur les discours visuels et les textes publiés dans ce domaine. Depuis la Grande Guerre, les photographies d'actualité ont été codifiées ; concrètement, leur intensité violente révèle la gravité d'un événement dans la conscience nationale ${ }^{2}$. En décortiquant ainsi les formes visuelles liées à la Guerre froide, nous serons à même de décider de sa place dans l'imaginaire collectif français. Nous verrons tout d'abord que les épisodes de Guerre froide, en 1962, sont présentés sous une forme plus ou moins anxiogène qui n'obéit pas à la chronologie des faits. Si, dans la réalité géopolitique, le premier semestre 1962 apparaît relativement calme tandis que le second met le monde au bord du gouffre avec la crise des fusées de Cuba, c’est exactement le schéma inverse qui domine la presse magazine où un premier semestre journalistiquement dramatisé précède un second semestre à la violence largement euphémisée. Ce paradoxe journalistique renvoie à la place secondaire de la Guerre froide dans les représentations françaises, simple prisme de lecture d'une actualité nationale bien plus essentielle.

\section{DES REPRÉSENTATIONS VISUELLES A CONTRARIO DES ÉVÉNEMENTS DE GUERRE FROIDE}

Curieusement, en 1962, la représentation violente de la Guerre froide est totalement déconnectée de l'ampleur réelle des événements.

Frappe tout d'abord le fait qu'au cours de l'année 1962, l'hebdomadaire crée une dramatisation conflictuelle sur la Guerre froide

2. Beurier Joëlle, Photographier la Grande Guerre France-Allemagne, Rennes, Presses universitaires de Rennes, 2014. 
alors que les relations politiques Est-Ouest apparaissent calmes. À plusieurs reprises, il fabrique un face-à-face entre les deux Grands, dont émanent des degrés variables de tension. Ainsi, au détour d'un reportage touristique sur le Danube, la légende d'un paysage idyllique rappelle la tension Est-Ouest en arrière-plan : « cette photo est une carte de géographie : ici commence le rideau de fer $»^{3}$. Plus étonnamment, sa veine people, censée incarner légèreté et superficialité, l'antithèse même de l'événement historique guerrier, est en prise sur la Guerre froide. Un sujet sur les stars de la vie internationale évoque comment « Un déjeuner chez Jackie a libéré Powers », Francis Gary Powers, le pilote du fameux avion Lockheed U-2 abattu le $1^{\text {er }}$ mai 1960 lors d'une reconnaissance clandestine au-dessus de l'URSS ${ }^{4}$. La photographie de gauche montre en pleine page deux femmes riant aux éclats, Mme Salinger, femme de "l'éminence grise de Kennedy », avec la fille de N. Khrouchtchev. Elle fait face au récit par Paul Mathias de l'échange des espions russe et américain. On assiste ainsi, d'un point de vue médiatique, à une "guerrefroidisation » des thèmes les plus anodins, introduisant la tension au cœur du divertissement géographique ou people des Français.

À un degré supérieur d'intensité, on relève également des propos nettement agressifs, voire guerriers, dans le domaine des avancées technologiques, principalement spatiales. Ainsi, le 3 mars 1962, le lancement en orbite autour de la terre de l'astronaute Glenn est présenté comme «la revanche de tout un peuple " sur l'URSS ${ }^{5}$. Les visages souriants, tendus vers le ciel, les mains jointes comme en prière du public en liesse, exprimeraient la victoire enfin remportée, la vengeance même sur l'adversaire : « une femme se jette à genoux sur le sable en disant : "ma prière est enfin exaucée”. À cette seconde, l'Amérique est tout entière débarrassée du “complexe de Gagarine” qui, depuis le 12 avril 1961, la hantait. Quand Glenn ajouta quelques minutes plus tard : "Quel spectacle! Tout est magnifique !”, chaque Américain sentit que le temps de la longue humiliation venait enfin de se terminer. " Si la course de l'espace fut réelle, le lecteur est en droit de supposer que les Américains photographiés expriment tout d'abord le soulagement et leur joie que leur compatriote n'ait pas explosé en vol, comme le racontent d'ailleurs les pages suivantes insistant sur les heures d'angoisse vécues par la famille de Glenn durant le vol. Si la course technologique est bien l'échelon inférieur d'une opposition Est-Ouest, le vocabulaire employé et la dramatisation iconographique affichée pour narrer la première mise en orbite, sont porteurs d'une violence contenue

3. Paris-Match, 10 février 1962.

4. Paris-Match, 17 février 1962.

5. Paris-Match, 3 mars 1962. 
bien supérieure à celle du reportage sur la crise des fusées, en novembre 1962. En mars 1962, les Soviétiques demeurent des adversaires.

Ils deviennent même des ennemis quand l'hebdomadaire invente, visuellement, un événement conflictuel qui n'existe pas. Le 28 avril 1962, deux reportages en double page présentent les ténors de la vie politique internationale. Le premier évoque tout d'abord le bloc communiste sinosoviétique, à travers les figures de Mao et de Khrouchtchev : « $\mathrm{K}$ a gagné : Mao admet qu'on peut éviter la guerre ", reproduisant les débats entre les frères communistes sur la nécessité ou pas de la coexistence pacifique avec l'Occident ; sur le même mode, la double page suivante poursuit la métaphore du conflit, dans le bloc capitaliste cette fois-ci, et montre John Kennedy debout et incisif, face à un homme assis et hésitant : " Kennedy triomphe aussi : il fait plier les rois de l'acier ${ }^{6}$. Le registre lexical est celui du combat : « victoire », « triomphe ». De même, le texte précise que le président américain se trouve sur «le porte-avion atomique le plus grand du monde » pour des exercices militaires, comme pour donner une tonalité militaire à ses propos économiques. Enfin, le parallélisme binaire de la syntaxe et de la typographie renforce l'illusion d'un face à face entre Khrouchtchev et Kennedy, qui ne renvoie pas à la réalité des faits rapportés par les articles : celle d'une opposition politique sino-soviétique d'une part, et celle d'une controverse économique interne aux États-Unis d'autre part. Au contraire, la succession des deux doubles pages liant apparemment les deux sujets - alors que les deux reportages n'entretiennent aucun rapport de sens - crée virtuellement l'illusion d'un face à face international de Guerre froide. Paris-Match fabrique donc un conflit larvé entre les deux Grands quand il n'existe pas. Par le vocabulaire et la maquette, il fabrique dans l'esprit du lecteur français, une opposition frontale et agressive inexistante. Ainsi, en 1962, l'information sur la Guerre froide est déconnectée de la réalité chronologique et l'hebdomadaire invente une intensité émotionnelle aux accents guerriers, sans prise réelle sur l'actualité.

Mais peut-être la confrontation inventée et la tension fabriquée ne relèvent-elles que d'une tactique de vente pour fabriquer du scoop ; une manière de créer de l'angoisse dans une stratégie commerciale fondée sur le sensationnel ? On pourrait le penser avec le reportage du 6 janvier 1962, où Paris-Match accumule arbitrairement les ingrédients de la dramatisation photojournalistique. Dans ce premier numéro de l'année, la Guerre froide apparaît dans le traumatisme des familles séparées par le récent mur de Berlin, construit en août $1961^{7}$. Le vocabulaire du titre est hyperbolique : « le défi de Noël devant le mur de la honte ». Il joue sur le registre des émo-

6. Paris-Match, 28 avril 1962.

7. Paris-Match, 6 janvier 1962. 
tions extrêmes, ici celui de l'affrontement et de la transgression morale. Les thématiques sont également puissantes, la double page suivante présentant le murage d'une fenêtre de Berlin-Est en gros plan, dont ne sortent plus en haut que la main du maçon et sa truelle, sans vie. En vis-à-vis, à l'ouest, un père grimpé sur un pilier exhibe son jeune fils à la famille restée de l'autre côté. Le vis-à-vis est constant derrière la thématique de l'emprisonnement et du traumatisme des séparations. Paris-Match fait le choix de clichés s'étalant en doubles ou en pleines pages. Les contrastes en noir et blanc sont fortement outrés, renforçant la tonalité douloureuse de l'information. La communication iconographique est renforcée par le pathos textuel : «De ces fenêtres de l'Est, on ne sautera plus, même pour mourir » ou bien " fugitif présent de Noël : le petit garçon montré à sa grand-mère, en face, à l'est ». Étonne ici le fait que Paris-Match ait fait le choix d'un numéro de nouvel an sur Berlin, sans lien naturel avec l'actualité immédiate, comme pour aller réveiller l'émotion pendant la trêve de Noël.

Cette mise en tension de certaines thématiques de la Guerre froide surprend d'autant plus qu'a contrario, l'événement le plus dangereux de la période, la crise des fusées qui dura du 14 au 28 octobre 1962, est présenté sous un jour totalement apaisé. Dans l'esprit de tous, politiques et ambassadeurs ou témoins épars qui ont suivi l'événement à la radio le souffle coupé, celle-ci reste le moment le plus fort de ces années. Pourtant, la couverture de Paris-Match est bien éloignée de ce climat d'une haletante intensité psychologique, et le numéro du 3 novembre 1962 relate plus une promenade militaire aux Caraïbes que le prélude d'une nouvelle guerre mondiale. Certes, le magazine évoque immédiatement l'événement comme l'antichambre d'une troisième guerre mondiale. L'importance qu'il lui accorde transparaît dans la longueur de l'article (vingt-neuf pages), en plus des titres clairement militaires des deux premières doubles pages: "Ces bateaux qui ont failli déclencher la guerre ", "L’île est bloquée. Pour la base US, c'est l'état de guerre ». L'hebdomadaire est ici en conformité avec l'angoisse qui a étreint les populations et le souvenir qui lui a fait suite. Mais la violence du climat psychologique de l'événement n'est pas relayée par l'atmosphère que véhiculent les images publiées par Paris-Match. Danger et menace du titre sont en effet rapidement contredits par l'évacuation de la tension, au plus haut sommet de l'État américain. Ainsi, deux clichés juxtaposés montrent le président Kennedy, tête baissée sur le micro, épongeant la sueur de son front, sa main cachant presque tout le visage (page de gauche) ; en regard, sur la page de droite, sous le désormais célèbre cliché aérien révélant la position des différents missiles, le président américain, de profil, éclate de rire dans un fauteuil confortable. La légende se fait même explicite, pour limiter l'impact angoissant de la première photographie de la tension extrême : «Kennedy : Il a pris la décision la plus 
grave de sa vie, puis soudain, il s'est détendu ». Le magazine choisit ainsi, tant sur le plan lexical que dans la mise en page iconographique, d'évacuer immédiatement la tension. Certes, le lecteur français est déjà informé de l'issue heureuse de la crise, dénouée quelques jours plus tôt. Toutefois, le lectorat du magazine n'est pas tenu plus de quelques secondes en empathie avec l'angoisse présidentielle, et la rapide évacuation du choc psychologique tranche avec le mode de communication habituel de Paris-Match.

De même, alors que les premiers titres portent la trace de la guerre, très peu d'images l'évoquent, et bien maladroitement. Aucun des topoï visuels de la violence de guerre, en vigueur depuis 1915, n’est ici présenté. Le cliché de la ruée des troupes sur un champ de bataille, quatre pages après le début du reportage, est intitulé « Cuba où commence l'affrontement des Américains et des Russes ». Malgré le titre évocateur, les troupes avancent, mais ne courent pas, les corps ne sont pas courbés comme dans une attaque réelle. Le lecteur n'est pas projeté photographiquement dans l'urgence d'une véritable attaque. Du reste, en déchiffrant le sous-titre, il apprend que l'image montre un entraînement à Guantanamo et bien évidemment pas la sortie des troupes qui n'a pas eu lieu. Le magazine propose ainsi quelques succédanés de photographies de guerre, mais bien peu convaincantes. De même, quelques pages plus loin, un canon rappelle qu'il peut tirer ; mais, photographié de face, devant des marines sereins, il se révèle au repos et sans danger réel. Plus clairement, à aucun moment de son reportage, l'hebdomadaire ne cherche à faire monter la tension chez le spectateur, par l'illusion visuelle de l'action que produisent photographiquement le mouvement, la fumée ou le chaos. Textuellement, jamais l'angoisse de la violence ou de la menace ne saisit le lecteur à la gorge.

Au contraire, Paris-Match publie des images de paix pour informer sur la plus grave crise de la Guerre froide. Les guetteurs cubains épiant « chaque geste des marines » sourient presque à l’objectif ; les principales troupes d'invasion sont de jeunes ingénieurs russes, musclés et bronzés, qui feuillettent des magazines en caleçon de bain, accompagnés de leurs épouses glamour qui s’adonnent à la photographie: «Dans les palaces de la Havane, les techniciens ont succédé aux milliardaires américains » du temps de Batista, sous une forme bien peu guerrière ${ }^{8}$.

Ce sont donc uniquement les images techniques qui vont véhiculer l'aspect militaire du conflit, reprenant en cela un topos visuel, permettant depuis le début du $\mathrm{Xx}^{\mathrm{e}}$ siècle, d'aseptiser la guerre ${ }^{9}$. Se racontent surtout

8. Paris-Match, 3 novembre 1962.

9. Bussemeyer Marianne, Deutscher illustrierte Presse: eine soziologischer Versuch, Heidelberg, 1930. 
les préparatifs «à bord du porte-avion atomique Enterprise » ${ }^{10}$, pour contrer un éventuel passage en force des navires russes : avions qui décollent, câbles qui pendent, pistes de décollage et casques de protection sur les oreilles, prière à l'heure fatidique, les clichés indiquent la préparation à la guerre à l'intérieur du bateau plus que la guerre elle-même. Les dernières pages du magazine, en couleurs, représentent alors la vie, l'animation, mais pas plus que les titres, les images ne reflètent la tension de l'angoisse. Comme dans tous les reportages de guerre, la focalisation sur les aspects techniques occulte la violence et évacue le danger d'une mort éventuelle.

Un tel reportage peut sembler conforme à la réalité de l'événement, non seulement dépassé et résolu à la date de publication, mais où pas une goutte de sang ne fut versée. Pourtant, alors que la marque de facture de Paris-Match repose sur l'intensification permanente des moindres sujets, en des périodes parfois déconnectées de toute tension réelle, le magazine refuse explicitement, pour l'événement le plus dangereux de la période, les jeux de maquettes, de contrastes, de formats, les titres sensationnels et les images provocantes qui constituent sa griffe. Cet étonnant refus de la dramatisation psychologique signale une décision éditoriale dont il faut comprendre le sens.

\section{D’AUTRES GUERRES PLUS IMPORTANTES}

Pour comprendre l'utilisation de la violence médiatique en 1962, il convient de s'interroger d'abord sur sa place habituelle dans Paris-Match, en particulier en ce qui concerne la représentation des guerres.

Paris-Match se caractérise justement par la recherche systématique de l'événement guerrier dans son actualité intérieure ou internationale. La violence y est déclinée sous diverses formes et à différents degrés. C'est même l'une des marques de fabrique de l'hebdomadaire, qui cherche systématiquement à propulser l'actualité dans une dimension historique, souvent conflictuelle ou morbide, pour fabriquer le sensationnel qui fait vendre. La place des événements armés y est donc quantitativement importante. Pas un numéro n'est publié sans un reportage illustrant une action violente. Il peut s'agir, en janvier 1962, d'un reportage au titre sans appel : « Nous avons assisté à la fin de Goa ». La décolonisation des Indes portugaises y apparaît dans des files de prisonniers encadrés par des soldats en armes ${ }^{11}$. Le reportage peut concerner également la révolte d'une prison brésilienne où les gardiens menacent de leurs armes les prisonniers couchés à terre. En 1962, la représentation des

10. Paris-Match, 3 novembre 1962, Une.

11. Paris-Match, 13 janvier 1962. 
événements violents oscille entre $17 \%$ et $50 \%$ des pages du numéro ${ }^{12}$. Armements, prisonniers, les topoï visuels de la guerre sont récurrents. Sans rentrer dans le détail des pratiques journalistiques qui favorisent la montée en tension des lecteurs, retenons que, de manière systématique, Paris-Match fait ses choux gras de l'événement conflictuel.

Les guerres françaises du $\mathrm{XX}^{\mathrm{e}}$ siècle sont ainsi particulièrement représentées. À partir du 2 juin 1962, la Seconde Guerre mondiale fait l’objet de cinq suppléments de seize pages narrant « les cinq journées qui ont décidé du sort de la guerre par Raymond Cartier ». Ces reportages sont factuels et épurés d'agressivité. Il n'en va pas de même du reportage du 12 mai 1962, illustrant les dernières audiences du jugement en appel d'Eichmann, avant son recours en grâce. L'article s'ouvre sur une double page en noir et blanc. À gauche, dans une photographie excessivement contrastée, des prisonniers derrière des barbelés s'agitent, tournés vers la page de droite, vers la photographie de la salle du procès ${ }^{13}$. Le topos du barbelé inflige un choc visuel au lecteur, évoquant l'univers concentrationnaire et la souffrance extrême. Il est renforcé par le titre, « Eichmann est-il digne du bourreau », qui induit, en même temps que l'idée de sa prochaine exécution, celle des souffrances extrêmes qu'il infligea aux déportés. La titraille renchérit dans la dramatisation, et créé un suspense vital : «Une voyante de Buenos Aires avait prédit à l'ancien SS qu'il ne survivrait pas à son cinquante sixième anniversaire. Une polémique s'élève entre les philosophes du monde entier sur le sort de celui qui fit exécuter six millions de Juifs " ; « Le temps se ratatine. Entre la potence et lui, il n'y a plus guère qu'une demi-douzaine d'audiences de la cour d'appel, suivies d'un éventuel recours en grâce auprès du chef de l'État. Le dernier mot - probablement un NON - lui laissera tout juste le temps de maudire son époque, son destin et peut-être ses juges » : le parti pris est clair et le magazine affiche sa position. Pourtant, il maintient le suspense en évoquant les tergiversations présentes et potentielles. Le contraste excessif du noir et blanc des images et des textes, le registre de l'hyperbole et l'utilisation de l'oxymore entre « bourreau » et « digne », inscrivent les péripéties du procès dans la grande Histoire. Par l'outrance visuelle, par l'exagération émotionnelle ainsi fabriquées, les lecteurs sont renvoyés brutalement dans l'événement traumatique encore récent. Par la violence du ton, des propos, des images, le magazine donne l'illusion aux Français d'assister à la suite de la guerre, presque en direct, par la mise à mort annoncée du coupable.

Le premier conflit mondial est également sollicité, et sa violence est rappelée à propos d’un fait divers brutal, l'explosion de la poudrière du

12. Respectivement pour les numéros du 3 février 1962 et du 2 juin 1962.

13. Paris-Match, 12 mai 1962. 
Banc-Rouge qui eut lieu le 9 avril 1962, en Ardèche. Celle-ci fut entendue à plus de 30 kilomètres à la ronde, tua dix-huit personnes, et traumatisa durablement la région. Deux semaines plus tard, le 21 avril 1962, ParisMatch reproduit, dans une double page en noir et blanc, un champ de vignes où les ceps alignés ressemblent à des croix blanches. Derrière s'élève une colonne de fumée grise. Dans sa partie haute, la double page suivante montre des hommes en capote et casque Adrian. Vus de dos, ils semblent se recueillir au-dessus des croix : « comme un champ de bataille, la terre de Saint Just d'Ardèche recèlera longtemps encore des pièges mortels : les obus et détonateurs qui n’ont pas explosé. Pendant que le village enterre ses morts, les soldats explorent les vignes pas à pas ». Dans la partie basse de la double page, des femmes, le fichu sur la tête, pleurent, collectivement. Tous les éléments iconographiques et textuels sont rassemblés pour provoquer la résurgence des images de la Première Guerre mondiale, des champs de croix aux soldats en uniformes, des larmes d'une société et d'un territoire, tout entier traumatisés par un événement aux traces durables. L'image des soldats parcourant le champ de vigne renvoie plus particulièrement à la scène du film d'Abel Gance, J'accuse, sorti en 1919, où ceux de 14 reviennent hanter les vivants pour leur rappeler leur devoir. Les liens entre les événements sont ténus et se limitent à la violence d'une explosion. Pourtant, les codes référentiels sont démultipliés et propulsent le fait divers de 1962 dans la violence de 14-18. Ce tissu référentiel rappelle autant la prégnance du devoir de mémoire envers ceux de $14^{14}$ qu'il met en évidence le remploi de topoï visuels renvoyant à la guerre totale, pour susciter l'émotion médiatique. Ainsi, la violence de guerre est construite, dans le magazine, pour évoquer des événements à l'importance factuelle toute relative, avec surtout une intensification émotionnelle qu'on ne retrouve pas pour l'événement majeur de la Guerre froide qu'est la crise des fusées. À côté de l'explosion de la poudrière ou du procès Eichmann, la crise de Cuba semble le théâtre d'un fait divers, presque un non-événement.

La guerre d'Algérie, enfin, fait l'objet d'une couverture particulièrement violente. À partir du 3 février 1962, la brutalité sanglante des attentats de l’OAS, perpétrés en métropole ou en Algérie, donne lieu à des images profondément choquantes. Semaine après semaine, affluent les clichés les plus terrifiants de la violence de guerre. Depuis la Grande Guerre, toute évocation visuelle d'un conflit passe par la publication de soldats dans l'action, d'armements, d'explosions, et même de corps déchiquetés, de paysages bouleversés et de représentations d'assaut. Identiquement à l'année 1915 où le tabou de la publication du corps - et plus fortement encore celui

14. Audoin-Rouzeau Stéphane, Becker Annette, 14-18, retrouver la guerre, Paris, Gallimard, 2000, p. 197-258. 
du corps français - est transgressé, en 1962, le magazine exhibe les détails choquants des affres de la guerre. Les dégâts matériels sont assénés dans leur crudité. Ainsi, le 17 février 1962, dans la chambre ravagée de la petite fille blessée dans l'attentat de l'OAS, le petit lit et le landau sont abandonnés sur un sol jonché de débris ${ }^{15}$. Un mois plus tard, le 17 mars, une double page exhibe le «no man's land d'Oran ${ }^{16}$. Les topoï visuels habituels disent la guerre : la rue vide, barrée de barbelés, où demeurent quelques objets abandonnés, illustre une ville assiégée. Les explosions, matérialisées par les débris fumants d'une voiture (24 mars) ${ }^{17}$ ou la violente lumière d'une explosion (10 mars) $)^{18}$, se multiplient. Plus brutalement, le corps abîmé est montré sans ambages. Le 3 février, il est tout d’abord blessé, comme le visage en sang d'une secrétaire du Quai d'Orsay où "l'explosion vient d'avoir lieu » ${ }^{19}$. Le 17 février, la blessure est plus profonde au visage de la blessée : l'œil, déchiqueté, n'est plus visible dans une bouillie sanguinolente qui dégouline sur son visage. Les cadavres abondent également. Ils gisent dans des flaques de sang, le 3 février ${ }^{20}$. Le 10 mars, ils sont simplement recouverts d'un journal ouvert ${ }^{21}$, d'un linceul le 24 mars, abandonnés dans la rue et que contemplent les passants : " c'est peut-être un parent, mais ils ne s'arrêtent plus » ${ }^{22}$. Les corps semblent dégingandés, comme dans le cliché d'un lambeau de pèlerine accroché dans un arbre, rappelant le cadavre soufflé des guetteurs des guerres passées (24 mars) ${ }^{23}$. Enfin, les familles sont éplorées et pleurent leurs morts ${ }^{24}$. Une nouvelle fois, ParisMatch réutilise les topoï les plus brutaux pour dire la violence de guerre et raconter la fin du dernier conflit colonial de la France.

La violence visuelle constitue donc l'arrière-plan culturel de l'année 1962, associée aussi bien aux événements militaires qu'aux faits divers. Pourtant, de la Guerre froide, la violence est pratiquement absente ; quand elle existe, elle n'est qu'une tension psychologique, déconnectée des événements réels. La violence visuelle ne serait-elle alors que la résultante du nombre des corps produits par l'événement? L’hypothèse séduit par son évidence, mais les chiffres la contredisent : toute actualité mortelle, voire morbide, n’engendre pas systématiquement la représentation de cadavres.

15. Paris-Match, 17 février 1962.

16. Paris-Match, 17 mars 1962.

17. Paris-Match, 24 mars 1962.

18. Paris-Match, 10 mars 1962,

19. Paris-Match, 3 février 1962.

20. Paris-Match, 3 février 1962.

21. Paris-Match, 10 mars 1962.

22. Paris-Match, 24 mars 1962.

23. Paris-Match, 24 mars 1962.

24. Paris-Match, 24 février 1962. 
Lors de l'explosion du Banc-Rouge, les corps éparpillés des dix-huit victimes ne furent que suggérés par la référence historique à 14-18, dont le public connaît alors parfaitement le caractère mutilatoire. Ainsi, la représentation sensationnelle de l'événement n'est pas proportionnelle à sa violence sur le terrain. Qu'est-ce qui explique alors l'étonnante couverture de la Guerre froide dans le magazine illustré ?

\section{GUERRE FROIDE ET CULTURE DE GUERRE CENTENAIRE}

En précisant la chronologie des dramatisations/apaisements dans la présentation médiatique de la Guerre froide, il ressort que ce n'est ni sa présence dans l'actualité internationale, ni le degré de violence que les événements recèlent, qui justifie son appropriation par les Français, via le magazine. L'actualité de la Guerre froide est en réalité lue et déformée par le prisme d'une culture de guerre nationale, profondément ancrée dans les mentalités.

En effet, de janvier à avril 1962, on trouve dans les différents reportages, un substrat de violence psychologique non négligeable. Comme nous l'avons montré, l'information la plus légère est associée à la Guerre froide et le magazine dramatise le sujet ; les succès spatiaux sont présentés sous le jour d'une compétition agressive entre les deux Grands; même les événements mineurs, et parfois malgré l'absence de lien entre les deux blocs, sont le prétexte à la fabrication d'une opposition marquée par un vocabulaire guerrier.

Durant cette période, la violence imprègne les sujets les plus anodins. L'éditorial du 6 janvier 1962 est ainsi intitulé « Neige et fascisme ». Il présente une information de saison qui porte sur les « champs de neige », les « vacances » et le « désir de respirer un peu d'air pur » à la montagne, dans un registre lexical explicitement guerrier, en total décalage avec le sujet: " Assiégés par la violence et encerclés par les propagandes contradictoires, les Français viennent d'opérer une sortie victorieuse qui leur a permis d'occuper leurs positions d'hiver sans d'autres pertes, traditionnelle, hélas ! des batailles routières de fin d'année ${ }^{25}$. De la même manière, les informations heureuses sont systématiquement associées à un épisode négatif qu'il eût été pourtant possible et simple d'occulter. Le 24 février 1962, ParisMatch couvre l'ouverture de la coupe du monde de ski à Chamonix. La Une fête la victoire d'un compatriote : « NOTRE COUVERTURE, Bozon, champion du monde : voir à l'intérieur notre reportage sur les drames et les triomphes français de Chamonix ${ }^{26}$. D’emblée, la titraille embraye non pas sur les

25. Paris-Match, 6 janvier 1962.

26. Paris-Match, 24 février 1962, Une. 
succès, mais sur ce qui vient les limiter, et consacre deux pages entières au drame de « la petite Marielle » Goitschel, arrivée deuxième dans la finale. En parallèle, les numéros de février, puis de mars, multiplient les images des attentats perpétrés en métropole comme en Algérie, sous les dehors les plus crus précédemment révélés, et l'ensemble de l'information du magazine est sombre et violent. Cette période correspond aux attentats perpétrés par l'OAS jusqu'au mois d'avril 1962, date où le référendum sur l’indépendance algérienne est approuvé à plus de 90 \% par la population métropolitaine et augure d'un processus de résolution de la violence.

En conséquence, de mai à décembre 1962, on assiste à la disparition des tensions de manière générale, et à la présentation d'une Guerre froide sous des dehors apaisés. Elle devient presque un non-événement, plus un fait divers social ou culturel qu'un événement historique conflictuel. Aux antipodes de la dramatisation antérieure, elle apparaît par exemple sous la forme d'une détente culturelle, voire d'une espèce de circulation d'hommes et d'influence entre les deux blocs. Le 5 mai 1962, les ballerines du Bolchoï sont comparées aux Girls du lido : « Décidément, il y a quelque chose de changé à Moscou » (première double page) ; «On dirait les Girls du Lido. Ce sont les étoiles de la chorégraphie soviétique » (deuxième double page) ${ }^{27}$. L’idée de la supériorité culturelle de l’Ouest y est évidente, mais elle s'exprime par l'humour et induit une circulation de l'Ouest vers l'Est, bien plus qu'une compétition ou un affrontement. Le 12 mai 1962, la circulation des ressortissants et des influences s'accentue ; les hommes semblent passer entre les deux blocs en toute liberté : une double page évoque «Un poète russe en liberté », c'est-à-dire un jeune écrivain soviétique passé à l'Ouest et riant aux éclats avec des jeunes écossais ${ }^{28}$. Quelques pages plus loin, « La coqueluche de Moscou : cette pianiste française » qui se promène tranquillement dans les rues de la capitale soviétique où elle vient passer un concours, sourit devant le mur où deux fois son portrait «a été découpé et retiré par des admirateurs anonymes ${ }^{29}$. Rires et sourires entre l'Est et l'Ouest conduisent alors au plus haut sommet de l'État, vers « ce virtuose qui a bouleversé K » dans le numéro du 2 juin $1962^{30}$.

Plus clairement encore que dans le domaine culturel, les épisodes liés à la conquête de l'espace reprennent un ton purement scientifique. Le 12 mai 1962, la «préparation du voyage sur la lune prévu pour 69 » donne lieu à une titraille neutre, sans photographies : « voici contrôlé par le grand savant Wernher von Braun le récit heure par heure du premier voyage 
terre/lune/terre effectué en 1970 par trois cosmonautes américains » ${ }^{31}$. Quelques semaines plus tard, la préparation de l'astronaute se focalise d'abord sur les transformations de la vie familiale: les enfants que l'on vient embrasser dans leur sommeil, la complicité du couple, révélant d'abord la tendresse et la sérénité familiale avant une efficacité virile dans le travail scientifique. Clairement, après avril 1962, tous les épisodes artistiques ou technologiques précédemment dramatisés du sceau de la Guerre froide, deviennent des faits divers internationaux, pacifiés et plaisants.

Ainsi, l'articulation chronologique autour de la résolution du conflit algérien domine les représentations sur la Guerre froide. Leur éventuelle dramatisation n'est pas connectée aux tensions internationales, mais semble bien plutôt liée à l'actualité nationale dense de l'année 1962, et à la fin douloureuse du conflit franco-algérien. La Guerre froide n'existe qu'à travers le prisme de l'actualité française. Quand la vie intérieure est douloureuse et tendue, en particulier à cause des attentats de l'OAS et durant toutes les négociations d’Évian, jusqu'à leur ratification lors du référendum du 8 avril 1962, l'ensemble de l'actualité est comme gangrené par le climat anxiogène qui sévit en France. La concurrence technologique se fait guerre larvée, et les relations Est-Ouest reviennent sur le devant de la scène, sans lien avec le réel, sous une forme globalement belliqueuse. En revanche, quand la vie française est en voie d'apaisement, la tension retombe progressivement au fil des numéros ; la conquête de l'espace redevient compétition technologique, sans vengeance ni violence ; les relations Est-Ouest se limitent à des circulations d'influence culturelle. Cette tournure pacifiée est telle que même un événement international aussi menaçant que la crise des fusées, n'est pas investi d'un potentiel menaçant. Dès lors, on peut dire que la Guerre froide, dans les représentations françaises, s'achève avec les accords d’Évian.

En 1962, la co-variation statistique entre la violence du conflit algérien et le reste de l'actualité révèle que la société française baigne dans un climat de « culture de guerre », dont la violence irrigue, par capillarité, tous les sujets de l'actualité ${ }^{32}$. La Guerre froide n'est qu'un sujet parmi d'autres, bien éloigné des tensions principales qui angoissent les Français : angoisse de la mort anonyme et aveugle, qui frappe en Algérie comme en métropole ; angoisse surtout face à l'avenir de la Nation, tout entière menacée d'implosion. "La France réduite à deux camps, voilà le danger ", titre Tournoux le 17 février 1962 en pleine période d'attentats, et il rappelle plus loin que «tout est en place pour l'affrontement». C'est le spectre de

31. Paris-Match, 12 mai 1962.

32. Pour une définition précise de la notion de culture de guerre, voir AudoinRouzeau S., Becker A., op. cit. 
la guerre civile qui connote d'anxiété toute l'information en France. La Guerre froide ne produira jamais une telle information angoissée dans la mesure où elle n’a jamais menacé de partition le corps national.

La Guerre froide apparaît donc, dans les représentations françaises, comme un événement secondaire. Soit elle est un épiphénomène presque ludique, cantonné à l'art, l'aéronautique ou la politique extérieure ; soit elle reste une variable de lecture, le prisme d'une culture de guerre éminemment française, qui se caractérise depuis près de cent ans par l'angoisse, ravivée encore une fois en 1962, de la partition du corps national. 